\title{
Implicaciones educativas del lenguaje LOGO
}

\section{Juan Ruiz}

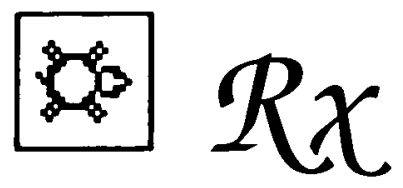

En este artículo se revisan diversas aportaciones sobre la utilización del lenguaje de programación LOGO en la escuela desde edades tempranas. Se plantea como las actividades con LOGO pueden ser utilizadas no sólo para el aprendizaje de conceptos y técnicas de programación, sino para el desarrollo de destrezas más generales y de estrategias cognitivas de exploración y descubrimiento, como alternativa a las rutinas memoristicas de aprendizaje.

\section{Introducción}

En la actualidad, los investigadores en el campo de la Educación han comprendido que la actividad de programación, además del interés que tiene como instrumento para la resolución de problemas de diversa índole, puede ser un poderoso recurso didáctico, no ya para el aprendizaje de conceptos y técnicas del campo específico de la Informática, sino para el desarrollo de destrezas más generales y valiosas de tipo heurístico (Palacios, 1987; Monteil, 1990; Núñez et al. 1991).

El tipo de problemas que los ordenadores pueden resolver está cambiando profundamente desde la aparición de los micro ordenadores interactivos que incorporan posibilidades de repre sentación gráfica, colores, sonidos, etc. Estos nuevos modelos hacen posible «plantear problemas» asequibles a niños cada vez más pequeños $y$, sobre todo, problemas que les atraen y que pertenecen a su mundo de intereses: juegos, dibujar un coche, una casa, flores, etc., de modo que al tiempo que realizan una actividad lúdica, manejan conceptos científicos complejos (Rodríguez Roselló, 1986; Arias et al. 1988; Díaz et al. 1989).

Estas nuevas posibilidades de los ordenadores son las que han llevado a realizar experiencias educativas sobre programación de ordenadores, en lenguajes apropiados, desde preescolar. El diseño del lenguaje LOGO ha estado ligado expresamente con la concepción de un lenguaje para experimentar y aprender, adaptándose fácilmente a diversos niveles de desarrollo de habilidades de pensamiento y favoreciendo formas exploratorias y estrategias de aprendizaje (García-Ramos y Ruiz, 1985).

\section{CARACTERISTICAS DEL LENGUAJE LOGO}

Un activo impulsor del empleo de los ordenadores controlados por los alumnos es Seymour Papert. Hacia mediados de los años sesenta comenzó a trabajar con un 
grupo de investigadores en el Instituto Tecnológico de Massachusetts para encontrar modos de uso de los ordenadores que facilitaran el aprendizaje a los niños; desarrollando, para dicho fin, un lenguaje de programación al mismo tiempo simple y potente: el LOGO.

Papert trabajó con Piaget en el Centro de Epistemología Genética de Ginebra. Esto le permitió comprender que los niños son constructores de sus propias estructuras intelectuales. Tienen un don innato para aprender; incluso antes de ir a la escuela acumulan un extenso saber: no aprenden sólamente a hablar, sino que también aprenden la geometría intuitiva para desplazarse en el espacio, aprenden bastante lógica e incluso retórica, sin que nadie les haya enseñado (Papert, 1.980).

¿Por qué, entonces, ciertos aprendizajes tienen lugar tan rápida y espontáneamente mientras que otros son retrasados e impuestos?. La respuesta que cabe dar es que el niño, constructor de su propio conocimiento, necesita «materiales» para ello. Precisamente, la función que Papert y sus seguidores atribuyen al ordenador es el de un instrumento didáctico que proporciona al niño modelos para facilitarle la adquisición de conceptos, principios, reglas y generalizaciones, que de otro modo se verían retrasados o incluso no se adquirirían.

Las dos ideas básicas del trabajo de Papert en sus investigaciones sobre el empleo de los ordenadores en la educación han sido:

- Es posible diseñar lenguajes de programación que los niños son capaces de aprender fácilmente y que les permiten hacer cosas de interés para ellos.

- Que esta actividad de comunicación de los niños con los ordenadores influye positivamente en su manera de pensar y en su manera de aprender otras cosas.

De estas ideas nace el lenguaje LOGO, uno de cuyos subsistemas, la "geometría de la tortuga» o «gráficos de la tortuga», es especialmente indicado para iniciar a los niños en un lenguaje de programación.

La tortuga es un «animal cibernético» - un pequeño triángulo - cuyos desplazamientos por la pantalla se pueden controlar. LOGO es el lenguaje de ordenador por medio del cual es posible comunicarse con la tortuga. La idea de programar se introduce por medio de la metáfora de enseñar a la tortuga nuevas palabras.

En el trabajo con la geometría de la tortuga existe un componente motivacional del máximo interés. Los chicos se sienten atraidos por esa actividad y utilizan, para desarrollar sus proyectos conceptos tales como: segmento, dirección, ángulo, medidas angulares, relaciones entre ángulos, figuras elementales, repetición controlada de un proceso, etc. Las representaciones geométricas que se consiguen pueden motivar largas exploraciones en las que el pensamiento numérico y el geométrico se entrelazan con la estética (Wat,1984; Díaz y Batanero, 1985; Abelson y Dissesa, 1986; Arias, 1989).

Como características generales del lenguaje logo podrían señalarse las siguientes (Rodríguez Roselló, 1986):

a) Logo es un lenguaje intérprete.

b) Logo es un lenguaje interactivo.

c) Logo es un lenguaje modular.

d) Logo es un lenguaje de micromundos.

e) Logo es un lenguaje recursivo.

LOGO es un lenguaje intérprete, pues admite las instrucciones en su propia terminología y las compila a lenguaje máquina para que el ordenador las ejecute. Pertenece a los lenguajes de alto nivel al igual que BASIC, PASCAL....

LOGO es un lenguaje interactivo, ya que, cualquier primitiva (instrucción predefinida en el propio intérprete) puede ejecutarse simplemente tecleándola, pasando así mismo a incorporarse al capírulo de primitivas todos los procedimientos 
definidos por el usuario. Por tanto LOGO se presenta como un lenguaje abierto a la creatividad del usuario para ampliar su propio potencial.

LOGO es un lenguaje modular, está pensado para ser escrito mediante procedimientos (agrupación de sentencias o comandos bajo un mismo nombre), los cuales pueden ser incorporados a otros procedimientos más complejos, simplemente llamándolos por dicho nombre. Esto proporciona una gran riqueza y versatilidad a la programación en LOGO.

LOGO es un lenguaje recursivo, esto supone la posibilidad de que un procedimiento se llame a sí mismo, lo que confiere una gran potencia a la programación.

LOGO es un lenguaje de micromundos, entendiéndose por estos los distintos entornos o ambientes en los que LOGO puede trabajar. Son programas que proporciona el maestro o el investigador en vistas a fines educacionales concretos, los cuales son difíciles o incluso imposibles de alcanzar con el logical Logo ordinario y comercialmente disponible.

Por ejemplo, al ser el desplazamiento de la tortuga casi instantáneo, en la mayoría de las versiones Logo, los investigadores de varios proyectos Logo han creado programas en los cuales la tortuga se mueve más lentamente cuando gira. Ello permite a los niños ver más claramente como se realizan los diferentes giros (Hillel \& Samurcay, 1985; Matos, 1986). Otros han programado a la tortuga de manera que deja una huella luminosa cuando gira (Zack, 1986). La huella de estos rayos permanece en la pantalla durante un pequeño periodo de tiempo, después de que la rotación se halla efectuado.

Algunos investigadores ofrecen inicialmente a los niños un conjunto de procedimientos especialmente preparados junto con -o en lugar de- las primitivas elementales que normalmente se encuentran disponibles en la geometría de la tortuga. En los estudios de Hughes, Macleod y Potts (1985), se les proporcionó a los alumnos procedimientos que dibujaban un cuadrado, triángulo, estrella, círculo además de las órdenes simples F(orward), B(ackwards), L(eft), R(ight) y H(ome). Mendelson (1986), Díaz et al. (1989), Ruiz et al. (1993), en esta misma línea, proponen la utilización de procedimientos definidos previamente por el profesor para la realización de figuras más complejas.

Otros investigadores se han preocupado de construir un entorno Logo totalmente nuevo, dirigido a proveer de medios naturales y convincentes para los que el lenguaje logo original no ofrecía una vía de acceso cómoda. Ejemplos de tales micromundos son los de Lohete (1986), sobre el movimiento en un espacio tridimensional, los micromundos de Dreyfus y Thomson (1986) para analizar acontecimientos probables.

Los entornos de aprendizaje con Logo son considerados por los expertos como uno de los aspectos fundamentales a tener en cuenta en las tareas de programación con este lenguaje (Papert, 1980; Leron, 1985; Littlefield et al. 1986; Resnick, 1987; King, 1990).

Según Papert (1980), las aptitudes en programación habrían de ser adquiridas según la estrategia del autodescubrimiento: el aprendizaje se daría de manera espontánea y natural, análoga a la de un niño pequeño cuando aprende a hablar. Papert lo llama "aprender sin que te enseñen". Este concepto del proceso aprender-enseñar se basa en la idea constructivista en la cúal los niños construyen y desarrollan sus propias estructuras intelectuales a través de la interacción con el entorno (De Corte y Verschaffel, 1987).

Las destrezas y conceptos que los niños pueden aprender con las actividades LOGO dependen, por una parte del tipo de micromundo utilizado (gráficos de tortuga, tortugas dinámicas y múltiples, proceso de listas, música, etc) y también del entorno de aprendizaje y del factor profesorado. Muchos de los efectos positivos previstos por Papert y otros investigadores, pueden quedar en una mera potencialidad si el niño no participa de un entorno apropiado (dos alumnos por ordenador y aulas reducidas de 15 a 20 alumnos ) y si el profesor carece de los conocimientos necesa- 
rios, tanto sobre el lenguaje como sobre la «filosofía educativa» que debe orientar su actuación (Díaz y Batanero, 1985; Leherer y Smith, 1986; Emihovich y Miller, 1986; Arias, 1988; Díaz et al. 1989).

Es necesario crear un clima en el aula que promueva una actitud relajada y constructiva hacia los errores, así como un ambiente de colaboración y ayuda mutua. El profesor debe estar atento a las necesidades de cada niño, proporcionando el tipo de ayuda apropiada en cada momento, pero sin que su intervención 777 anule un proceso de búsqueda y de descubrimiento personal (Leron, 1985).

El principal beneficio que se espera de LOGO, aparte de la adquisición de conocimientos propios del proceso de datos y los específicos sobre desarrollo de conceptos, serán de tipo más general y difíciles de evaluar: una actitud favorable hacia la escuela e interés por aprender, sentido de cooperaciòn con los compañeros y profesores, incremento en el concepto de sí mismo (sentido de destreza y poder personal), una actitud constructiva hacia los errores, buena voluntad para afrontar riesgos y experimentar soluciones posibles, etc. (Papert, 1980; Consejería de Educación Gobierno de Canarias, 1987; Arias, 1988).

\section{INVESTIGACIONES SOBRE UTILIZACION DEL LENGUAJE LOGO}

Las experiencias que en numerosos centros venían realizándose desde hace bastantes años sobre la introducción de los niños en la programación de LOGO, habían sido poco evaluadas, no llegando a ofrecer conclusiones que avalaran las supuestas ventajas que habrían de detectarse en los alumnos a partir de las actividades realizadas con LOGO. En los últimos años se vienen evaluando de forma más rigurosa algunos de estos temas a través de estudios en que se han utilizado diversas técnicas de recogida de datos tales como:

- Grabaciones en video, o las llamadas «dribble files» en las que se recogen todas las actuaciones de los niños mientras programan LOGO.

- Análisis de los proyectos y materiales escritos por los niños.

- Análisis de las actitudes de los niños respecto a LOGO.

- Entrevistas con los alumnos acerca de las maneras en que operan con el ordenador y la tortuga.

Los principales descubrimientos, De Corte, E.y Verschafell, L. (1.987), se refieren a los siguientes temas:

a) Deficiencia en los conceptos de programación.

b) Deficiencia en las estrategias de programación.

c) Diferencias en el estilo de programación.

\section{DEFICIENCIA EN LOS CONCEPTOS DE PROGRAMACION}

\section{Programación secuencial}

Un buen número de niños, aún después de trabajar bastantes horas con LOGO, tienden a desarrollar una programación secuencial («Spaghetti style») donde sólo utilizan órdenes primitivas tales como: AVANZA, GIRA, RETROCE$\mathrm{DE}$, sin llegar a utilizar conceptos más elevados como variables o recursividad (Leron, 1985; Mc Allister, 1986).

\section{Falta de lógica subyacente}

Otros alumnos emplean en la programación conceptos más elevados - variables, sentencias condicionales, recursividad- pero sin comprensión de la lógica sub- 
yacente. Copian un procedimiento, sin interesarse por la estructura y funcionamiento del procedimiento, a esta forma de operar se le llama "creación sin comprensión" (Leron, 1985; Burton \& Magliaro, 1986).

Estas investigaciones están en la línea del estudio de Leherer y Smith (1.986) acerca del nivel de conocimientos y comprensión de las nociones principales del lenguaje LOGO por parte de los niños. La dificultad para resolver problemas planteados en los que tenían que emplear procedimientos complicados era notable en todos los alumnos, a pesar de los distintos grados de instrucción. En aquellos casos en que tenían que emplear la recursividad, sólo el $21 \%$ entendió su uso.

\section{Deficiencia en las aptitudes de programación}

Dentro de este campo de investigación se pretende estudiar si los alumnos emplean métodos heurísticos tales como: planificación del problema, descomposición y depuración.

Gran cantidad de niños, mientras exploran con LOGO, no utilizan estrategias heurísticas propias de la resolución de problemas. Los resultados obtenidos se consiguen a menudo a través de una técnica de ensayo y error (trial and error).

Las dificultades de depuración son así mismo notables tal como han demostrado Mac Coy-Carver y Klahr (1.985). Niños de 7 a 9 años, tras un curso de LOGO de 24 horas no habían adquirido estrategias eficientes de depuración.

\section{Diferencias del estilo de programación}

Los niños presentan distintos estilos de programación, que pueden estar en la base de algunas dificultades detectadas en las tareas de programación. Una de estas dificultades radica en representar y descomponer figuras complejas en sus elementos constitutivos. Mc Dougall (1.986) señala como estas dificultades pueden estar debidas, en parte, a la inclinación de los niños a dibujar en primer lugar los perfiles y por su rechazo a repasar las líneas o dividir líneas rectas.

El estilo de programación puede estar tambien en función del tipo de proyecto en que se está trabajando. Según Sutherland y Hoyles (1.986) los niños tienden a percibir sus diseños bien como copias del mundo real u objetos abstractos, en el primer caso se realizan programas poco estructurados; en el segundo, al producir diseños más abstractos, se utilizan conceptos más sofisticados tales como variables, recursión etc.

La adquisición de un nivel tan bajo por parte de los niños en su capacidad de programación, según las investigaciones señaladas, puede estar condicionado por la corta duración de las experiencias en los estudios realizados. La programación es una habilidad compleja que requiere un buen número de horas de práctica, en la mayoría de las experiencias los niños sólo estuvieron un promedio de 20 a 40 horas trabajando con el ordenador (De Corte y Verschaffel, 1987).

La carencia de un tratamiento educacional adecuado, estaría en la base de los bajos niveles alcanzados por los niños en los estudios señalados (Leron, 1.985). Actualmente se tiende hacia un enfoque más directo y estructurado, donde la intervención del profesor va más allá de dar respuesta simplemente a la demanda de los alumnos.

Respecto a las estrategias de enseñanza, utilizadas en la programación con Logo, las experiencias realizadas por Littlefield, Delcros, Franks, Clayton y Bransford (1.986) sobre los efectos de diferentes métodos de enseñar - sin estructurar, estructurados y mediacionales - en la transferencia de capacidades de raciocinio a problemas non-LOGO, vienen a apoyar la tesis que aboga por una mayor estructuración en las tareas de enseñanza aprendizaje de LOGO. 
Los métodos no estructurados basados en el principio de aprender descubriendo, fueron comparados con los métodos estructurados donde se daban hojas a los niños con los trabajos a realizar comprobando si trabajan en las tareas asignadas.

Los métodos estructurados se mostraron más eficaces que los no estructurados en el dominio del LOGO, aunque no ofrecieron diferencias significativas sobre la transferencia non-LOGO.

Respecto a los métodos no estructurados y estructurados se compararon los métodos mediacionales, similares al enfoque estructurado, pero añadiendo la acción del maestro que intenta de forma explicita favorecer la transferencia, explicando a los alumnos las capacidades generales de razonamiento implicadas en las tareas así como la aplicación a situaciones non-LOGO. Los resultados obtenidos de esta comparación muestran a los métodos mediacionales como más eficaces respecto a los otros dos para lograr transferencias. No obstante ninguno de los tres se mostró efectivo para crear transferencias lejanas de capacidades de razonamiento (De Corte y Verschaffel, 1987).

La idea del autodescubrimiento como estrategia de enseñanza tiende a sustituirse por una acción más directa, por parte del profesor, a través de guías de estudio estructuradas, donde se «seleccionan» actividades a los alumnos o bien utilizando programas de LOGO especialmente diseñados, tales como los diferentes tipos de micromundos señalados, interviniendo de esta manera de forma más activa en el proceso de aprendizaje de los niños. Pero, no obstante, hay que señalar que pedir más estructuración no es lo mismo que alegar por el retorno al estilo de enseñanza tradicional, centrada en el maestro. De Corte y Verschaffel (1987) señalan los riesgos de organizar el entorno Logo demasiado minuciosamente, lo que pudiera dar lugar a todo tipo de efectos colaterales no deseables, como falta de motivación, alienación, miedo al fracaso, etc. Un entorno de aprendizaje potente es una situación en la cual «se encuentre el equilibrio entre dejar a los alumnos en libertad para trabajar en sus propios proyectos y la estructuración de la actividad para resultados específicos de aprendizaje». En esta misma línea se encuentran las aportaciones de Sutherland \& Hoyles (1986) y De Corte (1990).

Dentro del nuevo sistema educativo, donde se considera la posibilidad de utilizar diversas aplicaciones informáticas desde la educación primaria, el lenguaje Logo puede resultar un recurso valioso para la adquisición de destrezas y habilidades que faciliten aprendizajes posteriores.

En la utilización del lenguaje de programación Logo, consideramos que podrían investigarse el tipo de contenidos matemáticos, o de otras áreas del curriculum, suceptibles de ser tratados para su aprendizaje a través de este lenguaje de programación. En esta misma línea, sería interesante considerar la adquisición y desarrollo de estrategías para resolver problemas con ordenador, llegando a describir los procesos y mecanismos por los que los alumnos las adquieren y desarrollan.

Dentro de los nuevos diseños curriculares se plantea como un objetivo prioritario el «aprender a aprender», este objetivo ambicioso, pero al mismo tiempo irrenunciable, supone que los alumnos sean capaces de realizar aprendizajes significativos por sí solos en una amplia gama de situaciones y circunstancias. Desde esta perspectiva hay que señalar la importancia que debe otorgarse en el aprendizaje escolar a la adquisición de estrategias cognitivas de exploración y de descubrimiento, así como planificación y regulación de la propia actividad.

El ordenador, en función de su versatilidad, podría posibilitar el desarrollo de estrategías que favorezcan un aprendizaje más activo y exploratorio. Las posibilidades que ofrece el ordenador para la creación de entornos motivadores e individualizados, bien podrían ser utilizadas para la obtención de aprendizajes significativos, creando las condiciones idóneas para que se produzcan, teniendo en cuenta el estado de desarrollo del individuo y los esquemas actuales.

De forma más concreta, en algunas áreas se plantea la necesidad de desarrollar un conocimiento de procedimientos adaptables frente a la memorización de rutinas 
automatizadas. El ordenador puede ser una gran ayuda para el aprendizaje de tales procedimientos, ya que posibilita la creación de éstos y su manipulación. Determinar en que medida el ordenador puede propiciar estrategias de planificación y descubrimiento, que posibiliten aprendizajes significativos, es otro reto que se plantea ante la puesta en marcha del nuevo sistema educativo y la generalización en el uso de los nuevos recursos tecnológicos, y al que habrá que dar respuesta a través de fururas investigaciones.

\section{Referencias}

ABELSON, H., y DissesA, A. (1986). Geometría de la tortuga. El ordenador como medio de exploración de las Matemáticas. Madrid: Ed. Anaya.

ARIAS, J. M., y BELANGER, J.E. (1988). Manual de programación en Logo para la Enseñanza Básica. Madrid: Ed. Anaya Multimedia.

ARIAS, J. M. (1989). Logo: un entorno de trabajo. Apuntes de Educación y Nuevas Ternologias. 33, pp. 1-6.

BURTON, R. R., y BROWN, J. S. (1982). An investigation of computer coaching for informal learning activities. En D. Sleeman y J.S. Brown (Eds). Intelligent tutoring systems. 79-88. New York: Academic Press.

BURTON, J. K., y MAGLIARO, S. G. (1986). Computer programming and generalized problem solving skills: A critical review of the literature. Blacksburg: Virginia Polytechnic Institute and State University.

Consejeria de Educacion Gobierno de Canarias. (1987). Proyecto ABACO 85. Las Palmas de Gran Canaria.

De CORTE, E., y VersChaffel, L. (1987). Logo ¿Un medio para pen- sar? Logo y Educación. Feb. pp. 313.

De Corte, E. ; Verschaffel, L. ; SChrooten, H. ; Indemans, R., y Hoedemaekers, E. (1989). Logo as a vebicle for developing thinking skill in sixth graders. Comunication presented at the Tenth Biennial Meeting of the International Sociery for the estudy of vehavioural Development. Finland.

DE CORTE, E. (1990). Aprender en la escuela con las nuevas tecnologías de la información: Perspectivas desde la psicología del aprendizaje y de la instrucción. Comunicación, Lenguaje y Educación. $n^{\circ} 6$, pp. 93113.

DiAZ, J., y BATANERO, M. C. (1985). Microordenadores en la escuela. Madrid: Ed. Rama.

DiAZ, J.; BATANERO, M. C.; LOPEZ, L., y RUIZ, J. (1988). Logo para E. G.B. Zaragoza: Ed. Edelvives.

DiAZ, J.; BATANERO, M. C.; LOPEZ, L., y RUIZ, J. (1989). Logo para E.G. B. Libro del profesor. Zaragoza: Ed. Edelvives.

Diaz, J.; BatANero, M. C.; LopeZ, L., y Ruiz, J. (1990). LOGO para E.G.B. Ciclo Superior. Ed. Dpto. de Didáctica de las Matemáticas. Universidad de Granada.

Eminovich, C., y Milier, G.E. (1986). Talking to the turtle: A Discourse Analysis of Logo Instruction. Paper presented at the annual meeting of the American Educational Research Association. San Francisco.

GaRCIA-RAMOS, L., y RUIZ, F. (1985). Informática y educación. Panorama, aplicaciones y perspertivas. Barcelona: Ed. Garcia-Ramos.

HiLlel, J., y SAMURCaY, R. (1985). The definition and use of general procedeures bu 9 tear olds. En Hoyles, Cy Noss, R. (Eds). Procedings of the Logo and Mathematics Edu cation Conference. (pp. 88-96). Londres: Department of Mathematics, Statistics and computing,University of London.

KING, D. (1990). La aplicación del software en la Educación Especial. Comunicación Lenguaje y Educación. $n^{\circ} 5, \mathrm{pp} .31-47$.

LeHERER, R., y SMITH, P. C. (1986). LOGO Learning: Is Mose Better? Paper presented an the annual meeting of the annual mee ting of the American Educational Research Association, San Francisco.

LERON, V. (1985). The cognitive consequences of programming ins truction in class room. Educational Research. 14, pp.14-19.

Littlefield, J.; Delcros, V.; Franks, J. J.; Clayton, C., y Brans Ford, K. (1986). Some Prerequisites for Teaching Thinking: Some Lesson from a Study of LOGO Programming. Vanderbilt University, Nashville, Tennessee.

Matos, J. F. (1986). Constructing Logo procedures in drive mode. En Hoyles, R.; Noss, R. y Sutherland Eds. Proceding of the Second International Conference for Logo and Mathematics Education. pp. 233238. Londres: Department of Mathematics, Statistics and Computing. University of London.

MCALISTER, A. (1986). Abstraction and Logo programming strategies. (Student service project. Bulletin 17) Toronto: Toronto Board of Education.

MCCOY-CARvER, S., y KLAHR, D. (1985). Children's acquisition of debugging skills in a Logo environment. (Internal report) Pittsburgh: Department of Psychology, Camegie-Mellon Universicy.

MCDOUGALL, A. (1986). Children's dificulties in perceiving estructure and using subprocedures. En C. Hoyles; R. Noss y R. Sutherland (Eds). Proceedings of the Second Internation nal Conference for Logo and 
Matbematics Education. 30-37. London: Department of Mathematics, Statistics and Computing, University of London.

MONTEII, M. G. (1990). Primeros pasos en Logo. Madrid: Ed. Paraninfo.

NuÑEz, A.; Garcia, F.; Collado, J., y HernandeZ, S. (1991). LOGO. Aprender a pensar. Madrid: Ed. Paraninfo.

PALACIOS, J. (1987). Los micromundos son medios, no fines en si mismos. IV Seminario LOGO. Barcelona, 214-219.

PAPERT, S. (1980). Mindstorms: children, computers and powerful ideas. Brighton Harvester Press.

PAPERT, S. (1981). Desafio a la mente. Computadoras y educación. Buenos Aires: Ed. Galápago.

PAPERT, S. (1983). Enseñar a los niños a ser matemáticos versus enseñar matemáticas a los niños. En $P_{s i}$ cología genética y aprendizajes escolares. Ed. Madrid: Siglo XXI, 129-148.

RESNICK, L. B. (1982). Learning in school aut out. Educational Researcher. 16 (9), 13-20.

RODRIGUEz ROSELIO, L. (1986). LOGO: De la tortuga a la inteligencia artificial. Madrid: Ed. Vector Ediciones.

RodRiguez Rosello, L. (1988). Logo y curriculum. En Aguirregabiria, M. (Coor.) Tecnología y Educación. Ed. Narcea, 187-194.

Ruiz, J; LoPez, L. M.; Batanero, C., y Diaz, J. (1993). LOGO para Educación Secundaria. Consejería de Educación de la Junta de Andalucía. CEP de Jaén.

SUTHERLAND, R., y HoYles, C. (1986). Mathematics and Logo. a deve lopments in our intervention strategy. En C. Hoyles; R. Noss y R, Sutherland (Eds). Proceedings of the second In ternational Coference for Logo and Mathematics Education. 262-269. Londres: Department of Mathematics, Statistics an Computing. University of London.

ZACK, V. (1986). A study of ten to eleven years old students' notion of right angle in Logo geometry. En C. Hoyles; R. Noss y R, Sutherland (Eds). Proceedings of the second In ternational Coference for Logo and Mathematics Education. 262-269. Londres: Department of Mathematics, Statistics an computing. University of London.

\section{Implicaciones educativas del lenguaje LOGO Juan Ruiz$$
\text { CL\&E, 1994, 21, pp. 111-118 }
$$

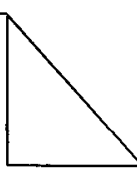

Resumen: En este artículo se revisan diversas aportaciones sobre la utilización del lenguaje de programación LOGO en la escuela desde edades tempranas. Se plantea como las actividades con LOGO pueden ser utilizadas no sólo para el aprendizaje de conceptos y técnicas de programación, sino para el desarrollo de destrezas mas generales. Desde una perspectiva constructivista del aprendizaje la utilización de este lenguaje de programación puede ser util para el desarrollo de estrategias cognitivas de exploración y descubrimiento, así como de procedimientos adaptables frente a rutinas memorísticas de aprendizaje.

Dirección: Facultad de Humanidades y Ciencias de la Educación. Departamento de Pedagogía. C/ Virgen de la Cabeza 4, 23071. Jaén

Datos del autor: Juan Ruiz, Doctor en Ciencias de la Educación, es Profesor de la Universidad de Jaén, Departamento de Pedagogía, área de conocimiento: Métodos de Investigación y Diagnóstico en Educación (M.I.D.E.)

(C) PERMISOS PARA CITAR O REPRODUCIR EN OTRAS FUENTES: Se pueden citar libremente hasta 500 palabras. Para reproducir una porción de texto mayor, figuras o ilustraciones, se deberá pedir permiso por escrito a la revista, especificando el uso al que se destina el texto. En todos los casos, se deberá citar el copyright de $C L \& E$. En el caso de artículos o textos que hayan sido a su vez reproducidos en $C L \& E$ los interesados deberán dirigirse tanto a los detentadores del copyright original como a $C L \& E$, en el caso de que se quiera hacer uso de la traducción. FOTOCOPIAS: Para todo lo relacionado con el uso mediante forocopia del material de esta revista, deberán dirigirse a: CEDRO, C/ José Marañón, 10, $3 .^{\circ}$ Izda. Tel. 5941575 . Fax 4453567. 


\section{LIBROS RECIBIDOS}

Acosta, V. et al (Ed.)

Programas de evaluación e intervención en educación especial. 1993.

Ajuntament de Barcelona

SELLARES, R. (Com.): La atención de las diferencias en la escuela. 1993.

Ajuntament de Plama

VV.AA.: Curs Palma Ciutat educativa.

Alfar

BORREgo, C.: Curriculum y desarrollo Socio-personal. 1992.

\section{Anthropos}

Esteva, C.: Cultura, sociedad y personalidad. 1993.

FIERro, A.: Para una ciencia del sujeto. Investigación de la persona (lidad). 1993.

TOLCHINSKY, L.: Aprendizaje del lenguaje escrito. Procesos evolutivos e implicaciones didácticas. 1993.

Ariel

TRILlA, J.: La educación fuera de la escuela. Ambitos no formales y edcuación social. 1993.

Gobierno de Canarias

VV.AA.: Memoria estadística de educación de adultos en la comunidad canaria. Curso 91-92. 1993.

\section{Grao}

VV.AA.: Com treballar els continguts procedimentals a l'aula. 1993.

ICE Universitat de Barcelona / Horsori

BorRego, C. (Coor.): Módulos didácticos. 2. Aprender a vivir juntos en la escuela. 1992.

Del Rio, M.J.: Psicopedagogía de la lengua oral: un enfoque comunicativo. 1993.

MEC / Morata

HICKs, D. (Comp.): Educación para la paz. 1993. PluCKRose, H.: Enseñanza y aprendizaje de la bistoria. 1993.

\section{Morata}

ELLотт: El cambio educativo desde la investigaciónacción. 1993.

\section{Morata / Paideia}

FERNANDEZ, M.: La profesión docente y la comunidad escolar: crónica de un desencuentro. 1993.

LISTON, D. P., y ZEICHNER, K. M.: Formación del profesorado y condiciones sociales de la escolarización. 1993.

\section{Paidotribo}

CHAVARRIA, X.: La educación física en la enseñanza primaria: del diseño curricular base a la programación de las sesiones. 1993.

VV.AA.: La educación física en primaria. Reforma. de 6 a 12 años. Guía del profesor. Volumen I y II. 1993.

\section{Pirámide}

Botella, C., y Benedito, M. C.: Asma bronquial. Evaluación e intervención en niños y jôvenes. 1993.

\section{S.M.}

VV.AA.: Programación y didáctica. Conocimiento del medio. 1992.

V.AA.: Programación y didáctica. Lengua. 1992.

Trillas

DALE, P. S.: Desarrollo del lenguaje. Un enfoque psicolingiuístico. 1992.

Diamondstone, J. M.: Talleres para padres $y$ maestros. Diseño, conducción y evaluación. 1991.

GORDILLO, J.: Lo que el niño enseña al hombre. 1992.

MORENO, S.: Guia del aprendizaje participativo. Orientación para estudiantes y maestros. 1993.

RODRIGUES, A.: Aplicaciones de la psicología social. 1988.

Waisburd, G., y SefCHovich, G.: Expresión plástica y creatividad. Guía didáctica para maestros. 1993. 